\title{
DNA deaminases: AIDing hormones in immunity and cancer
}

\author{
Svend K. Petersen-Mahrt • Heather A. Coker • \\ Siim Pauklin
}

Received: 11 May 2009/Revised: 3 June 2009 /Accepted: 8 June 2009 /Published online: 25 June 2009

(C) The Author(s) 2009. This article is published with open access at Springerlink.com

\begin{abstract}
It is well established that hormones can cause cancer, much less known is how they induce this change in our somatic cells. This review highlights the recent finding that estrogen can exert its DNA-damaging potential by directly activating DNA deaminases. This recently discovered class of proteins deaminate cytosine to uracil in DNA, and are essential enzymes in the immune system. The enhanced production of a given DNA deaminase, induced by estrogen, can lead not only to a more active immune response, but also to an increase in mutations and oncogenic translocations. Identifying the direct molecular link between estrogen and a mutation event provides us with new targets for studying and possibly inhibiting the pathological side-effects of estrogen.
\end{abstract}

Keywords Hormones · DNA mutations · Cancer · AID · APOBEC $3 \cdot$ Autoimmunity

\section{Introduction}

In 1938, the first cheap synthetic estrogen-like chemical (diethylstilbestrol) [1] was made available to patients, first

S. K. Petersen-Mahrt $(\triangle) \cdot H$. A. Coker $\cdot$ S. Pauklin

DNA Editing Laboratory, Clare Hall Laboratories,

London Research Institute,

South Mimms EN6 3LD, UK

e-mail: skpm@cancer.org.uk

Present Address:

H. A. Coker

Developmental Epigenetics, Department of Biochemistry,

University of Oxford,

South Parks Road,

Oxford OX1 3QU, UK as an experimental drug, then a few years later with FDA approval. In previous decades, the benefits of estrogen as a supposed remedy for a variety of diseases provided fertile ground for subsequent pharmaceutical exploitations. Yet, even as early as the late 1800 s, clinicians observed a link between hormones and cancer [2, 3], and in 1932, controlled laboratory research showed that estrogen can induce breast cancers [4]. Surprisingly, over the last 75 years, and despite the subsequent tragedy that resulted from the diethylstilbestrol, the molecular mechanism behind how estrogen exerts its oncogenic effects remained mostly elusive. Tests, including the Ames mutagenesis assay, were unable to show any significant effect of estrogen as a DNA-damaging agent. This led to the prevailing conclusion that estrogeninduced cancer through its ability to activate cell proliferation. More recently, other theories have tried to explain the oncogenic potential of estrogen, they included: inhibition of apoptosis, inactivation of DNA repair, and radicals from oxidative stress or metabolic derivatives of estrogen (if present at high concentrations) forming DNA adducts (reviewed in [5-7]). Although each theory addresses a particular aspect of estrogen's oncogenic potential, none are fully satisfactory in explaining how physiological amounts of estrogen can be oncogenic.

In 2002, we discovered a novel molecular mechanism that explained how antibody genes were diversified after antigen encounter during a humoral immune response [8]. Although known to shuttle between the nucleus and the cytoplasm, activation-induced deaminase (AID) is a predominantly cytoplasmic enzyme that, upon activation, enters the nucleus and is targeted to the immunoglobulin (Ig) locus. The enzyme then deaminates cytosine bases (dC) to uracil (dU) in single-stranded DNA (Fig. 1A; for reviews please see [9-11]). The protein itself had been identified a few years earlier, as an essential gene for antibody 
diversification (i.e. class switching (CSR) and somatic hypermutation (SHM)), and was named AID [12, 13]. Usually the DNA lesion (either as $\mathrm{dU}$ or as a $\mathrm{dU}: \mathrm{dG}$ mismatch) is faithfully repaired back to cytosine (Fig. 1B), but in the Ig locus it leads to DNA mutations and DNA recombination (Fig. 1C \& D). During SHM, single point mutations are introduced into the DNA of the Ig locus, which manifest themselves as, and are selected for, amino acid changes in the antigen-binding site. Targeting the constant region of the Ig locus with AID during CSR, allows for homologous DNA regions to recombine and loop out the intervening sequence, thereby placing a different constant domain into the active Ig locus. This change allows for the antibody to obtain a different effector function (e.g., from a predominantly membrane-bound IgM to a soluble IgE). The hallmark for both events is a natural protein-inducing DNA damage that does not lead to DNA repair, but to DNA alterations in somatic cells.

Although a number of AID's functions are unique to antibody diversification, further analysis has shown that our genome contains a family of DNA deaminases, some of which are active anti-viral agents - the AID evolutionary descendant APOBEC3 family of proteins [14-16]. APOBEC3G's DNA deamination activity is known to inhibit HIV replication, thought to be by inactivating the firststrand cDNA of the HIV genome during viral replication.

The discovery of DNA deaminases showed for the first time, that naturally occurring unaltered enzymes could mutate the DNA of somatic cells. This was in direct contrast to the dogma that somatic cells should maintain a stable genome, and made these enzymes obvious candidate oncogenes. AID's oncogenicity was demonstrated when overexpression of AID in transgenic mice caused tumor formation in different tissues (lung [17], lymphatic [17], and liver [18]). More recently, it was shown that AID was required for the $c-m y c / \operatorname{IgH}$ translocations of Burkitt lymphomas [19-21]. As shown in Fig. 1E, during this translocation $c$-myc's promoter recombines with the Ig locus, causing oncogenic expression of the $c-m y c$ gene. Aside from c-myc [22], it has been known that the process of SHM (or AID) targeting can occur outside the Ig locus [23, 24], leading to DNA-deaminase-induced mutation in tumor suppressors or proto-oncogenes [25]. Importantly though, the oncogenicity and DNA mutability of the DNA deaminases are not due to a genetic defect in the proteins, but rather a consequence of their normal (albeit misregulated) function. With the potential to mutate our genomes on a daily basis, understanding how AID is regulated will have a direct impact on immunity, immune pathologies, and cancer.

In our recent publication [26], we were able show that estrogen could stimulate the production of AID in primary B cells. Although primary mature B cells are differentiated and the AID locus may be 'available' (in terms of chromatin status), we did not require any co-stimulatory signals to detect the estrogen enhancement. We also confirmed that the increased production in mRNA showed a concomitant increase in AID protein. Furthermore, we observed a direct correlation with estrogen treatment, AID mRNA production and AID function in Ig class switching, and somatic hypermutation. Interestingly, the most significant correlation occurred between estrogen treatment and the number of potentially oncogenic $c-m y c /$ Ig translocations. We also observed this estrogen-induced response with most of the APOBEC 3s, indicating that the response to hormones is ancient and conserved.

In terms of molecular mechanisms, we were able to demonstrate that the cellular estrogen receptor (ER) is bound to the AID promoter after estrogen treatment. This seems to recruit a transcription activator complex, and thereby drive the transcription of the AID gene. Interestingly, when we co-stimulated the cells both with estrogen and through the NFKB pathway, we observed an enhanced binding of NFKB to the promoter (known to activate AID mRNA production [27]), and enhanced AID mRNA production, which was greater than from either stimulus alone. This may have immune consequences, in that during an immune reaction, additional stimulatory signals provided by estrogen would enhance AID production, leading to a potential hyperactive humoral immune response.

There is a large body of evidence that females can have a more pronounced immune reaction upon infection, which has also been observed at the level of Ig production [28, 29]. This gender bias is also seen in immune pathologies that are dependent on antibodies, such as systemic lupus erythematosus (SLE) [30, 31], an autoimmune disease that is ten times more prevalent in women than in men. Therefore, the ability of estrogen to enhance or even hyper-activate AID production, could partially explain the molecular mechanisms of certain autoimmune pathologies, via AID's role in antibody maturation.

Although men have estrogen as part of their endocrine system, the levels are much reduced compared to women. In terms of overall immune-fitness though, there does not appear to be a strong bias of men vs. women. Why then, is a potentially highly oncogenic protein under the control of such a panpleiotropic hormone? One can only speculate on the evolutionary origins, but a plausible hypothesis is that AID (or its ancestor) was a genome guardian against foreign DNA (as mentioned in $[8,10,14])$. The enzyme could act on a mobile DNA element and inactivate it through deamination/ mutation. This is partially substantiated by the following observations: (1) AID can partially inhibit retroviral infections [32]; (2) the APOBEC3 proteins predominately act to inhibit retroviral infections and retroelement mobility [14, $15,33]$; and (3) the Ig gene and its recombination potential 


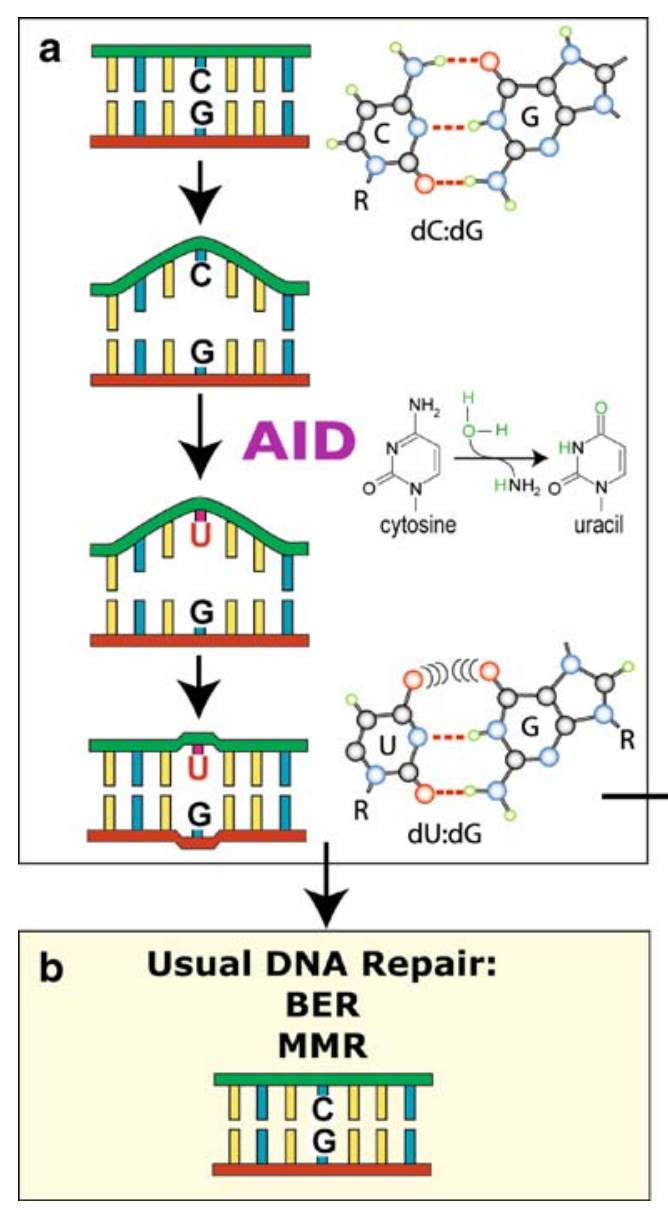

Fig. 1 The function and consequence of AID. (A) AID uses hydrolytic deamination to form uracil in single-stranded DNA, thereby inducing a twofold lesion in the DNA, an unusual base and a base mismatch. $(B)$ Normally, various DNA repair pathways repair the

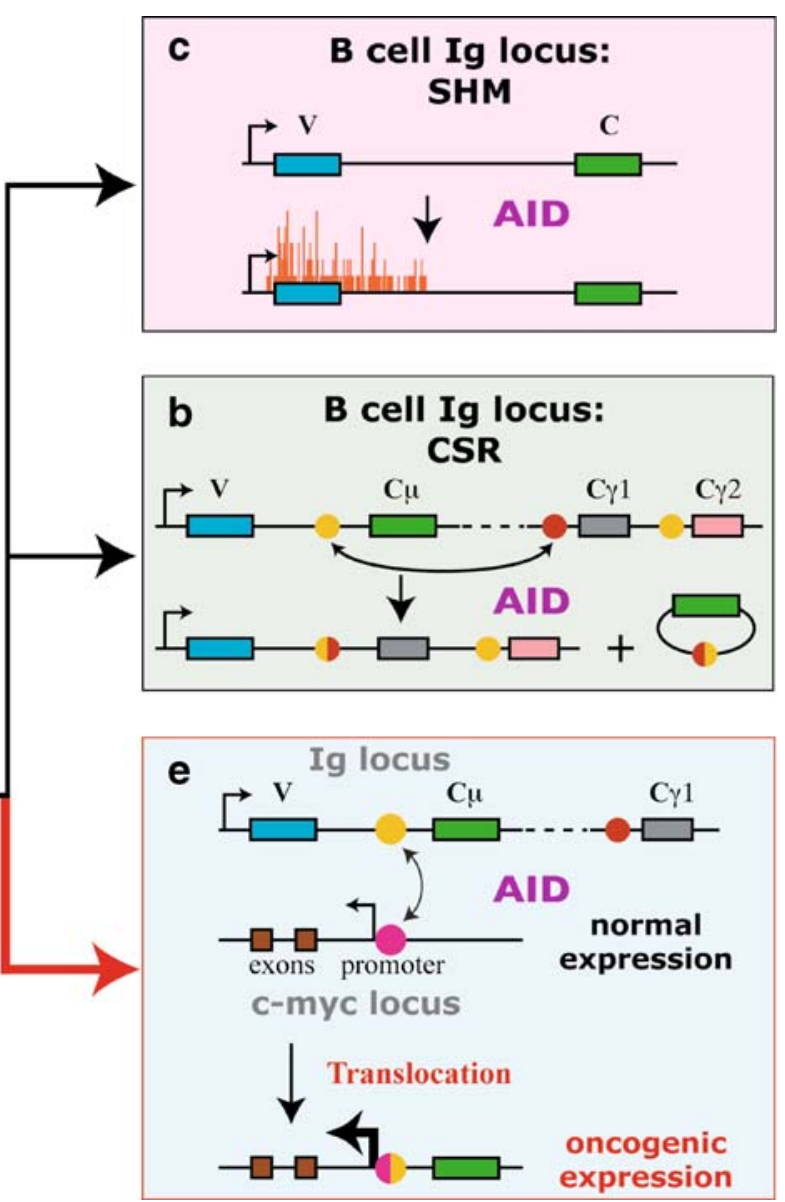

lesion back to cytosine. $(C)$ and $(D)$ At the immunoglobulin locus, the AID-induced lesion leads to SHM and CSR. (E) AID can induce oncogenic translocations during CSR, if the c-myc promoter rearranges into the Ig locus rather than another Ig constant region was evolutionary derived from a retroelement [34, 35]thereby linking the activity of AID to an invading DNA. As a genome guardian, it would be advantageous to have expression during the most vulnerable stage for retroelement infection of development - the unfertilized oocyte [36]. Already 5 years ago, we were able to show that AID is expressed in isolated mouse oocytes and embryonic germ cells [37]. In our current work [26], estrogen was able to increase AID mRNA in ovarian-derived primary cells by over 20 -fold. This provides good evidence that AID-induced deamination is not limited to B cells. In line with a more global response of AID expression due to estrogen, analysis of cell lines derived from breast, ovarian, placental, and prostate tissue, also showed that AID was upregulated in response to estrogen. Analogously, the other DNA deaminases (APOBEC3 members) were also activated in various tissues and cell lines after estrogen treatment.

It is not fully understood how AID, or its evolutionary descendants, can inactivate foreign DNA. Aside from

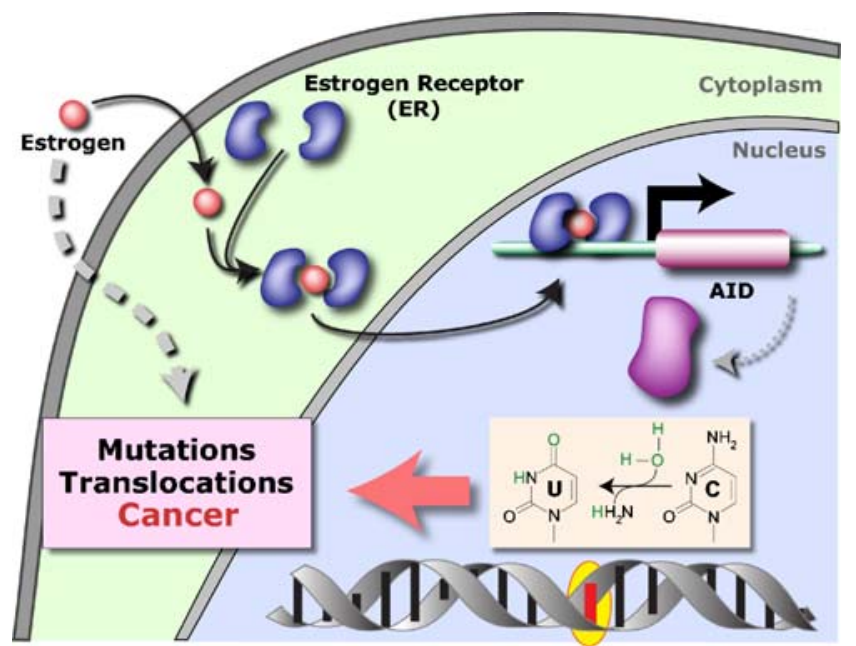

Fig. 2 Model of how estrogen induces DNA damage. Estrogen enters the cell and is bound by the ER. The dimeric ER-estrogen complex enters the nucleus and induces AID expression. This leads to an increase in mutations and translocations, and potentially cancer 
mutating cytosines in mobile elements in our genomes, an intriguing additional possibility is via deamination of 5methyl-cytosine $(5 \mathrm{meC}) .5 \mathrm{meCs}$ in the context of CpGs usually inactivate the mobility of DNA elements [36], but some elements are resistant to this mark. We have already demonstrated that AID can deaminate 5meCpG [37], and recent genetic evidence has linked AID to CpG DNA demethylation [38]. It is therefore possible that estrogendriven mutation of $\mathrm{CpGs}$ during oncogenesis may also be due to aberrant AID expression.

After more than a century, our current model (Fig. 2) may have brought us a step closer in understanding how estrogen can directly induce mutations leading to cancer in higher vertebrates. We suggest that endogenous or exogenous estrogen is absorbed by the target cell. In the cytoplasm, the dormant estrogen receptor (ER) will bind to the ligand and dimerizes. These structural alterations allow the receptor-ligand complex to enter the nucleus and target estrogen responsive genes. At the promoter of the DNA deaminase gene AID, the ER-estrogen complex recruits proteins for mRNA transcription. The enhanced AID message is then translated into increased amounts of AID protein. Excessive AID enters the nucleus and begins to deaminate cytosine bases to uracil. If these DNA lesions are not repaired, the consequence is mutation and translocations - leading to autoimmunity or oncogenesis. As the model predicts, the activity of estrogen is not dependent on cell replication, occurs in cells that are estrogen responsive, and does not require any a priori genetic mutations for manifestation.

Acknowledgment The authors wish to thank members of the DNA Editing Laboratory for support and discussions on work in the laboratory. We also apologize for not being able to include (or accidental omitting) important references from the various disciplines of hormones, immunity, autoimmunity, and cancer. This work was supported by Cancer Research UK.

Disclosure statement The authors declare that they have no conflict of interests.

Open Access This article is distributed under the terms of the Creative Commons Attribution Noncommercial License which permits any noncommercial use, distribution, and reproduction in any medium, provided the original author(s) and source are credited.

\section{References}

1. Dodds EC, Goldberg L, Lawson W, Robinson R (1938) Oestrogenic activity of certain synthetic compounds. Nature 141:247-248

2. Schinzinger A (1889) Über carcinoma mammae. 18th Congress of the German Society for Surgery Hirschwald, Berlin, pp 55-56

3. Beatson CT (1896) On treatment of inoperable cases of carcinoma of the mamma: suggestions for a new method of treatment with illustrative cases. Lancet 2:104-107
4. Lacasagne A (1932) Apparition de cancers de la mamelle chez la souris male, soumise a des injections de falleculine. Compt Rend Acad d Sc 195:630-632

5. Roy D, Liehr JG (1999) Estrogen, DNA damage and mutations. Mutation res 424:107-115

6. Cavalieri EL, Rogan EG (2004) A unifying mechanism in the initiation of cancer and other diseases by catechol quinones. Ann N Y Acad Sci 1028:247-257

7. Russo J, Russo IH (2006) The role of estrogen in the initiation of breast cancer. J Steroid Biochem Mol Biol 102:89-96

8. Petersen-Mahrt SK, Harris RS, Neuberger MS (2002) AID mutates E. coli suggesting a DNA deamination mechanism for antibody diversification. Nature 418:99-103

9. Neuberger MS, Harris RS, Di Noia J, Petersen-Mahrt SK (2003) Immunity through DNA deamination. Trends Biochem Sci 28:305-312

10. Petersen-Mahrt S (2005) DNA deamination in immunity. Immunol Rev 203:80-97

11. Di Noia JM, Neuberger MS (2007) Molecular mechanisms of antibody somatic hypermutation. Ann rev Biochem 76:1-22

12. Muramatsu M, Kinoshita K, Fagarasan S, Yamada S, Shinkai Y, Honjo T (2000) Class switch recombination and hypermutation require activation-induced cytidine deaminase (AID), a potential RNA editing enzyme. Cell 102:553-563

13. Revy P, Muto T, Levy Y, Geissmann F, Plebani A, Sanal O, Catalan N, Forveille M, Dufourcq-Labelouse R, Gennery A, Tezcan I, Ersoy F, Kayserili H, Ugazio AG, Brousse N, Muramatsu M, Notarangelo LD, Kinoshita K, Honjo T, Fischer A, Durandy A (2000) Activation-induced cytidine deaminase (AID) deficiency causes the autosomal recessive form of the Hyper-IgM syndrome (HIGM2). Cell 102:565-575

14. Conticello SG, Thomas CJ, Petersen-Mahrt SK, Neuberger MS (2005) Evolution of the AID/APOBEC family of polynucleotide (deoxy)cytidine deaminases. Mol Biol Evol 22:367-377

15. Harris RS, Liddament MT (2004) Retroviral restriction by APOBEC proteins. Nat Rev Immunol 4:868-877

16. Chiu YL, Greene WC (2008) The APOBEC3 cytidine deaminases: an innate defensive network opposing exogenous retroviruses and endogenous retroelements. Annu Rev Immunol 26:317-353

17. Okazaki IM, Hiai H, Kakazu N, Yamada S, Muramatsu M, Kinoshita K, Honjo T (2003) Constitutive expression of AID leads to tumorigenesis. J Exp Med 197:1173-1181

18. Endo Y, Marusawa H, Kinoshita K, Morisawa T, Sakurai T, Okazaki IM, Watashi K, Shimotohno K, Honjo T, Chiba T (2007) Expression of activation-induced cytidine deaminase in human hepatocytes via NF-kappaB signaling. Oncogene 26:5587-5595

19. Pasqualucci L, Guglielmino R, Houldsworth J, Mohr J, Aoufouchi S, Polakiewicz R, Chaganti RS, Dalla-Favera R (2004) Expression of the AID protein in normal and neoplastic B cells. Blood 104:3318-3325

20. Ramiro AR, Jankovic M, Eisenreich T, Difilippantonio S, ChenKiang S, Muramatsu M, Honjo T, Nussenzweig A, Nussenzweig MC (2004) AID is required for c-myc/IgH chromosome translocations in vivo. Cell 118:431-438

21. Robbiani DF, Bothmer A, Callen E, Reina-San-Martin B, Dorsett Y, Difilippantonio S, Bolland DJ, Chen HT, Corcoran AE, Nussenzweig A, Nussenzweig MC (2008) AID is required for the chromosomal breaks in c-myc that lead to c-myc/IgH translocations. Cell 135:1028-1038

22. Rabbitts TH, Forster A, Hamlyn P, Baer R (1984) Effect of somatic mutation within translocated c-myc genes in Burkitt's lymphoma. Nature 309:592-597

23. Muschen M, Re D, Jungnickel B, Diehl V, Rajewsky K, Kuppers $\mathrm{R}$ (2000) Somatic mutation of the CD95 gene in human B cells as 
a side-effect of the germinal center reaction. J Exp Med 192:1833-1840

24. Kuppers R, Dalla-Favera R (2001) Mechanisms of chromosomal translocations in B cell lymphomas. Oncogene 20:5580-5594

25. Liu M, Duke JL, Richter DJ, Vinuesa CG, Goodnow CC, Kleinstein SH, Schatz DG (2008) Two levels of protection for the $\mathrm{B}$ cell genome during somatic hypermutation. Nature 451:841-845

26. Pauklin S, Sernandez IV, Bachmann G, Ramiro AR, PetersenMahrt SK (2009) Estrogen directly activates AID transcription and function. J Exp Med 206:99-111

27. Dedeoglu F, Horwitz B, Chaudhuri J, Alt FW, Geha RS (2004) Induction of activation-induced cytidine deaminase gene expression by IL-4 and CD40 ligation is dependent on STAT6 and NFkappaB. Int Immunol 16:395-404

28. Butterworth M, McClellan B, Allansmith M (1967) Influence of sex in immunoglobulin levels. Nature 214:1224-1225

29. Eidinger D, Garrett TJ (1972) Studies of the regulatory effects of the sex hormones on antibody formation and stem cell differentiation. J Exp Med 136:1098-1116

30. Whitacre CC (2001) Sex differences in autoimmune disease. Nat Immunol 2:777-780

31. Cohen-Solal JF, Jeganathan V, Grimaldi CM, Peeva E, Diamond B (2006) Sex hormones and SLE: influencing the fate of autoreactive B cells. Curr Top Microbiol Immunol 305:67-88
32. Gourzi P, Leonova T, Papavasiliou FN (2006) A role for activation-induced cytidine deaminase in the host response against a transforming retrovirus. Immunity 24:779-786

33. Babbage G, Ottensmeier CH, Blaydes J, Stevenson FK, Sahota SS (2006) Immunoglobulin heavy chain locus events and expression of activation-induced cytidine deaminase in epithelial breast cancer cell lines. Cancer Res 66:3996-4000

34. Agrawal A, Eastman QM, Schatz DG (1998) Transposition mediated by RAG1 and RAG2 and its implications for the evolution of the immune system. Nature 394:744-751

35. Gellert M, Hesse JE, Hiom K, Melek M, Modesti M, Paull TT, Ramsden DA, van Gent DC (1999) V(D)J recombination: links to transposition and double-strand break repair. Cold Spring Harb Symp Quant Biol 64:161-167

36. Bestor TH (1999) Sex brings transposons and genomes into conflict. Genetica 107:289-295

37. Morgan HD, Dean W, Coker HA, Reik W, Petersen-Mahrt SK (2004) Activation-induced cytidine deaminase deaminates 5methylcytosine in DNA and is expressed in pluripotent tissues: implications for epigenetic reprogrammin. J Biol Chem 279:52353-52360

38. Rai K, Huggins IJ, James SR, Karpf AR, Jones DA, Cairns BR (2008) DNA demethylation in zebrafish involves the coupling of a deaminase, a glycosylase, and gadd45. Cell 135:1201-1212 